\title{
Una nueva figura profesional: el Community Manager
}

\author{
A new professional profile: the Community Manager \\ Dra. Araceli Castelló Martínez \\ Profesora Asociada Universidad de Alicante (UA) \\ Universidad Cardenal Herrera-CEU (UCH-CEU) \\ araceli.castello@ua.es - araceli.castello@uch.ceu.es
}

Recibido: 30 de Mayo de 2010

Aceptado: 20 de Agosto de 2010

\begin{abstract}
Resumen
La implantación en la sociedad de los espacios de la Web 2.0 y su empleo por parte de los anunciantes en sus estrategias empresariales han motivado la aparición de una nueva figura profesional: el Community Manager. La importancia del tema de estudio radica en la actualidad y novedad de la figura profesional analizada, dada la rápida incorporación que está viviendo como profesional de la comunicación online de la mano de los continuos cambios que estamos viviendo en el ámbito de la comunicación digital. El objetivo del presente artículo es profundizar en el perfil profesional de esta figura, en su origen y en el proceso de incorporación al mercado publicitario que está viviendo. La metodología se ha basado en un análisis de contenidos de la Web relacionados con esta figura, que ha dado como resultados una amplia variedad de contenidos sobre el Community Manager, así como en una entrevista auto-administrada con responsables de la Asociación Española de Responsables de Comunidad Online. Como conclusión, podemos decir que este profesional es cada vez más demandado por las empresas, entendido como aquella persona responsable de sostener las relaciones de ésta con sus clientes en el ámbito digital, en base al conocimiento de los planteamientos estratégicos de la organización y los intereses de los clientes.
\end{abstract}

\section{Abstract}

The introduction of Web 2.0 tools in the society and their employment by advertisers in their business strategies have led to the emergence of a new professional figure: the Community Manager. The importance of this subject of study is due to the current relevance of this professional figure, taking into account its fast incorporation to the advertising market as an expert in digital communication. This article aims to study in depth the professional profile of this figure, its origin and process of incorporation to the advertising market. The methodology has been based on an analysis of Web contents related to the Community Manager, as well as a self-administrated interview with persons responsible for the Spanish Association of Heads of Online Communication (AERCO). As a conclusion, we can say that this professional 
profile is increasingly demanded by companies, as the person responsible for supporting the relations with their customers in the digital world, thanks to the knowledge of both, the companies business strategies and the customers interests.

Palabras Clave: Comunicación, Internet, Publicidad, E-comercio, Relaciones Sociales, Blog.

Key Words: Communication, Internet, Advertising, E-commerce, Social Relations, Blog.

Sumario: 1. Introducción. 2. Método. 3. Marco teórico. 3.1. Estrategias empresariales en la Web 2.0. 3.2. Definición y funciones del Community Manager. 3.3. Perfil profesional del Community Manager. 4. Resultados. 5. Conclusiones. 6. Bibliografía. 7. Notas

Summary: 1. Introduction. 2. Method. 3. Theoretical Framework. 3.1. Business strategies in the Web 2.0. 3.2. Community Manager definition and functions. 3.3. Community Manager professional profile. 4. Results. 5. Conclusions. 6. Bibliography. 7. Notes.

\section{Introducción}

Con una audiencia mensual de más de la mitad de la población española y una inversión publicitaria de casi el $12 \%{ }^{1}$, Internet se ha convertido para muchas empresas en el medio idóneo para personalizar su mensaje, interactuar con el destinatario y satisfacer así nuestras expectativas como clientes. Afinidad con el público objetivo, generación de notoriedad con costes reducidos, segmentación y medición en tiempo real son algunas de las ventajas del medio más alabadas por los anunciantes.

Compartir, comunicar, conversar y cooperar son las $4 \mathrm{Cs}$ de la Web $2.0^{2}$, que por medio de espacios y herramientas como $\operatorname{los} b^{\circ} \log s^{3}$, las redes sociales online ${ }^{4}$, las plataformas de video

\footnotetext{
1 Internet cuenta con una penetración del 51,3\% de la población española, según datos de la oleada febrero-marzo de 2010 del Estudio General de Medios (EGM), elaborado por la Asociación para la Investigación de Medios de Comunicación (AIMC) y representa el 11,7\% en el reparto de la inversión publicitaria, ocupando el tercer lugar tras la televisión (41,2\%) y la prensa $(20,9 \%)$, de acuerdo con datos del "Estudio InfoAdex de la inversión publicitaria en España 2010" publicado por InfoAdex. Ambos estudios están disponibles en www.aimc.es y www.infoadex.es, respectivamente.

${ }^{2}$ Web 2.0 es un concepto acuñado por Tim O’Reilly, que lo define en su nivel más alto como un conjunto de aplicaciones que tratan de abarcar la red entendiendo cómo funcionan los efectos de red y aprovechándolos en todo lo que se hace. Se trata de todo aquello que se centra en explotar al máximo la participación y la información generada por consumidores.

${ }^{3}$ Se conoce como blogosfera al espacio virtual formado por blogs (o bitácoras, en castellano). Un Weblog (abreviado como blog) es una jerarquía de textos, imágenes, objetos multimedia y datos, ordenados cronológicamente, soportados por un sistema de distribución de contenidos capaz de proporcionar al autor la funcionalidad necesaria para distribuir esos contenidos con cierta frecuencia, exigiéndole unas capacidades técnicas mínimas y que puede facilitar la construcción de conexiones sociales significativas o comunidades virtuales alrededor de cualquier tema de interés. En muchas ocasiones es un "espacio individual para la comunicación" (Estalella, 2007: 20-37), actualizado periódicamente, en el que lo que se escribe está destinado a los demás. Las entradas o textos publicados por el autor, que el lector puede comentar, se llaman
}

Facultad de Ciencias de la Información - Universidad de La Laguna

Avenida César Manrique, s/n; Campus de Guajara

38071 La Laguna, Tenerife (Islas Canarias - España) 

sindicación $^{9}$ o el microblogging ${ }^{10}$, ha permitido al usuario generar contenidos, editarlos en base a sus intereses y compartirlos con su comunidad. Desde el lado de la empresa, escuchar y relacionarse con los clientes a través de estos nuevos espacios supone una transformación total de la cultura y la comunicación empresariales.

posts, y se presentan en orden cronológico inverso. Se considera que el primer weblog fue la página What new in 92, creada por Tim-Berners Lee en 1992 para divulgar las novedades del proyecto World Wide Web. Más tarde, 1997, sería Jorn Barger quien acuñaría el término, para referirse a su página web Robot Wisdom. Por blogger se conoce a la persona que regenta un blog. Fotologs, vlogs (o videoblogs), audioblogs y moblogs son algunas variantes del weblog, con características similares en cuanto a organización y diseño, en función del tipo de contenido que se comparta en el sitio web, a saber: fotos, videos, archivos de audio o contenido proporcionado desde teléfonos móviles, respectivamente.

${ }^{4}$ Una red social es un grupo de personas que tienen algo en común y es por ese algo por lo que se conocen y se agrupan para interactuar y estar en contacto. Según datos de Nielsen NetView, Facebook, Tuenti y MySpace son las redes más utilizadas en España. Para Celaya (2008: 92), una comunidad virtual puede definirse como:

"un grupo de personas que tienen un interés común y que desean relacionarse a través de la Red para compartir sus experiencias y opiniones sobre esa materia. Al igual que en el mundo analógico, una comunidad virtual suele tener una estructura, unas personas que la gestionan, una organización interna, un lazo que las une, etc., mientras que en una red social los temas publicados y los vínculos entre los usuarios son infinitos. (...) La mayoría de las comunidades virtuales pueden definirse también como algún tipo de red social, pero no todas las redes sociales virtuales forman necesariamente una comunidad".

${ }^{5}$ La más conocida es Youtube, fundada en febrero de 2005 por Hurley, Chen y Karim. Permite a los usuarios incluir, compartir y comentar videos. Es propiedad de Google, desde su compra en octubre de 2006 por 1.650 millones de dólares. Según datos de Youtube (www.youtube.com), la plataforma cuenta con más de 200 millones de usuarios únicos al mes, y es el sexto website más visitado en todo el mundo.

6 En ellas los usuarios pueden enviar y votar noticias, como en Menéame (www.meneame.net) o Culturízame (www.culturizame.net).

${ }^{7}$ Sitios web colaborativos que pueden ser editados y modificados por los usuarios. La palabra "Wiki" fue inventada por Ward Cunningham en 1995 a partir del término hawaiano "wikiwiki", que significa "rápido". En 2001, Jimno Wales y Larry Sanger desarrollaron la wiki más conocida hasta el momento, la Wikipedia (www.wikipedia.org), enciclopedia en la que los términos son introducidos y modificados por los propios internautas. Un ejemplo de wiki creada por una empresa es www.wikirecuerdos.com, una "enciclopedia nostálgica" creada por Bimbo en relación con algunos de sus productos de bollería en la que los usuarios pueden incluir y comentar recuerdos de su infancia.

${ }^{8}$ Los marcadores sociales, los sistemas de etiquetado y los de filtrado social permiten almacenar, clasificar y compartir enlaces a contenidos de Internet de forma sencilla. Todas ellas se basan en los llamados tags o etiquetas, palabras asociadas a una página web, foto o video que permiten identificar contenido y acotar su búsqueda, estableciendo incluso relaciones entre las personas que las emplean (Del.icio.us para páginas web y Flickr, para fotos, son ejemplos de marcadores sociales). Ejemplo de su uso con fines corporativos es la galería con que cuenta LG en Flickr (www.flickr.com/photos/lgblog).

${ }^{9}$ Permite a los usuarios suscribirse a los contenidos de cuantas fuentes deseen, de tal forma que tengan en un solo lugar, conocido como agregador, todo aquello que les interese. La fuente de sindicación más aceptada es RSS (Really Simple Sindication - Rich Site Summary). A la sindicación de archivos de sonido se le conoce como podcasting, término formado por el acrónimo de publico $n$ demand y la palabra broadcasting (retransmisión bajo demanda).

\footnotetext{
${ }^{10}$ Herramientas de comunicación que permiten a los usuarios enviar mensajes de texto con una longitud determinada de caracteres (en general, en torno a 140 caracteres) de forma instantánea y gratuita, como Twitter, Jaiku, Pownce o YouAre. Con sus cerca de dos millones y medio de usuarios, Twitter se ha convertido en el líder indiscutible del microblogging (Celaya, 2008: 209-218).
} 
España es el primer país europeo y el segundo del mundo, tras Brasil, en porcentaje de internautas que participan en una de las principales herramientas de la Web 2.0: las redes sociales online ${ }^{11}$. Con casi 500 millones de usuarios, Facebook es a día de hoy la red social de más éxito ${ }^{12}$, también entre las empresas, seguida de otras como MySpace, Tuenti o Linkedln.

Estos espacios permiten a las empresas participar en conversaciones y aportar contenido de interés para los usuarios, en un entorno favorable tanto para consumidores como para marcas. La creación de comunidades en torno a la marca en espacios como las redes sociales online facilita la construcción de branding, además de servir como canal de fidelización.

Este marketing dialogante e interactivo obliga a la organización, ahora más que nunca, a escuchar primero y vender después, en una clara orientación estratégica hacia el cliente. En este panorama, la investigación cobra todavía más importancia si cabe para la búsqueda de la eficacia en la comunicación a través de mensajes personalizados y relaciones individualizadas: la información sobre el cliente se convierte en el valor estratégico que va a marcar las diferencias en la gestión de las organizaciones ${ }^{13}$. Igualmente, para poder aprovechar todo el potencial de estas plataformas es necesario que la empresa gestione los valores de marca y construya la identidad corporativa de forma integral y holística.

Además, los usuarios compartimos nuestras experiencias como consumidores y tenemos en cuenta las opiniones y recomendaciones de nuestros allegados en nuestras decisiones de compra y consumo. La viralidad y la prescripción han cobrado protagonismo en la Web 2.0, como demuestran muchos estudios al afirmar que en nuestra interacción con otras personas a través de plataformas como Facebook o Twitter hablamos en muchas ocasiones de marcas. Por ejemplo, en la plataforma de microblogging Twitter el $20 \%$ de los tweets contiene referencias a alguna marca, según un informe independiente efectuado por Virgin Media Business ${ }^{14}$.

\footnotetext{
${ }^{11}$ Fuente: Nielsen (www.nielsen.com).

${ }^{12}$ Fuente: Facebook (www.facebook.com).

${ }^{13}$ Puede obtenerse más información sobre la orientación empresarial hacia el cliente en la Web 2.0 en: Castelló Martínez, A. (2010): "La orientación empresarial hacia el cliente en la Web 2.0", en Miguel Hernández Communication Journal 1, España, Mayo. Recuperado el 26 de mayo de 2010 de: http://mhcj.es/2010/05/12/castello/

${ }^{14}$ Fuente: www.virginmediabusiness.co.uk. Además, en Twitter el 48\% de los usuarios españoles sigue perfiles de marca, el $70 \%$ considera que las empresas deben dar información sobre ofertas y promociones en este espacio social y el $32 \%$ valora Twitter como un medio social idóneo para la comunicación corporativa. El 35\% de los encuestados en este estudio realizado por Addoor aseguró que la presencia de las marcas en Twitter mejoraba su imagen porque favorecía la cercanía y el $25 \%$ opinó que daba la sensación de que la empresa estaba a la última.
}

Afirma Best (2007: 20) que "existen muchas formas de medir el compromiso psicológico que tiene un cliente con una marca, pero el hecho de que la recomiende a otras personas se sitúa en el nivel más alto de vinculación emocional”.

Facultad de Ciencias de la Información - Universidad de La Laguna

Avenida César Manrique, s/n; Campus de Guajara

38071 La Laguna, Tenerife (Islas Canarias - España) 
De ahí la importancia de que la empresa vele por su reputación online con inmediatez y transparencia, utilice un lenguaje cercano, ofrezca contenido relevante y gestione su comunidad online de forma profesional. La figura del Community Manager es cada vez más demandada por las empresas, como aquella persona responsable de sostener las relaciones de la empresa con sus clientes en el ámbito digital, en base al conocimiento de los planteamientos estratégicos de la organización y los intereses de los clientes. Debe ser, así, la voz de la empresa puertas afuera y la voz del cliente puertas adentro, como destaca Connie Besson ${ }^{15}$.

En definitiva, los espacios de la Web 2.0 son cada día más utilizados por las organizaciones como soportes en los que llevar a cabo sus estrategias de marketing y publicidad ${ }^{16}$, al permitir a la empresa basada en las relaciones conversar con sus clientes actuales y potenciales. El consumidor se coloca, de este modo, en el centro de la relación con las marcas y, ahora más que nunca, de él depende el éxito empresarial.

\section{Método}

La implantación en la sociedad de los espacios de la Web 2.0 y su empleo por parte de los anunciantes en sus estrategias empresariales han motivado la aparición de una nueva figura profesional: el Community Manager. La importancia del tema de estudio radica en la actualidad y novedad de la figura profesional analizada, dada la rápida incorporación que está viviendo como profesional de la comunicación online de la mano de los continuos cambios que estamos viviendo en el ámbito de la comunicación digital.

De acuerdo con el estudio Nielsen Global Survey realizado por Nielsen Online en un total de 47 países, incluido España, la fuente de información de producto más fiable para los consumidores son las propias opiniones de otros usuarios, según declara el $78 \%$ de los entrevistados. Puede leerse la nota de prensa del estudio en: http://es.nielsen.com/news/20071023.shtml Para el estudio "El fenómeno de las redes sociales. Percepción, usos y publicidad" presentado por Zed Digital en noviembre de 2008, la recomendación de los amigos es lo que más confianza ofrece a los internautas para tener contacto con una marca (64\%). Si a esto se le une que el $67 \%$ de ellos afirma hablar de marcas con otras personas, Internet se convierte en uno de los canales que más influye en las decisiones de compra (la cifra asciende al 74\% para los usuarios de redes sociales online). Zed Digital también presentó en abril de 2007 el "Estudio sobre uso, interés, conocimiento y percepción de la blogosfera española”, en el que el $44,1 \%$ de los encuestados admitía estar dispuesto a cambiar su marca habitual por un comentario negativo leído en un blog (de hecho, un $41 \%$ afirmaba haber dejado de comprar algún producto por este motivo). Ambos estudios están disponibles en www.zeddigital.es

Otros estudios han demostrado que los usuarios son mucho más proclives a entrar en un anuncio cuando ven que sus amigos son seguidores de esa marca/campaña (Nielsen y Facebook) o que fans y seguidores de las marcas en las redes sociales se sienten más inclinados a comprar las marcas de las que son fans (Chandwick Martin Bailey-iModerate y Emarketer).

15 Extraído de: "La función del Community Manager", documento elaborado por AERCO (Asociación Española de Responsables de Comunidad Online) y Territorio Creativo en noviembre de 2009 y disponible en: http://www.box.net/shared/pgur4btexi

${ }^{16}$ Para más información sobre la viralidad, la orientación empresarial hacia el cliente, la segmentación y el branding en Internet puede consultarse: Castelló Martínez, A. (2010), Estrategias empresariales en la Web 2.0. Las redes sociales online. Alicante: ECU 
El objetivo del presente artículo es profundizar en el perfil profesional de esta figura, en su origen y en el proceso de incorporación al mercado publicitario que está viviendo. La hipótesis de partida destaca al Community Manager como un profesional demandado en la actualidad por las empresas anunciantes y cuyo perfil se está integrando en las estructuras y organigramas de agencias de publicidad y agencias de medios.

Para alcanzar los objetivos fijados y demostrar las hipótesis de trabajo se han empleado tres técnicas que podemos enmarcar dentro de la investigación cualitativa por la naturaleza de los datos obtenidos y el análisis que se ha llevado a cabo de los mismos: investigación documental, análisis de contenidos de la Web 2.0 relacionados con la figura del Community Manager y una encuesta auto-administrada con tres preguntas abiertas a responsables de AERCO (Asociación Española de Responsables de Comunidad Online).

Con respecto a la investigación documental, se han consultado diferentes tipos de publicaciones desde $1995^{17}$ a la actualidad en base a unas palabras clave definidas con el fin de localizar la información ya publicada sobre el tema de estudio, establecer un marco de referencia al fenómeno objeto de estudio y realizar un análisis histórico de la materia. Las fuentes consultadas han sido:

- Páginas web verticales especializadas en marketing y/o comunicación publicitaria, como www.puromarketing.com, www.marketingdirecto.com o www.marketingnews.es.

- artículos de revistas científicas y publicaciones periódicas especializadas en marketing, comunicación y/o publicidad relacionados con la materia de estudio, como Control, Interactiva digital, IP Mark, Brandlife, Anuncios, Estrategias de comunicación y marketing y El Publicista.

- Estudios y publicaciones de organismos y asociaciones del mercado publicitario, como Interactive Advertising Bureau Spain (IAB Spain), Asociación para la Investigación de Medios de Comunicación (AIMC), InfoAdex y European Interactive Advertising Bureau (EIAA).

El análisis de contenidos de la Web 2.0 relacionados con la figura profesional que nos ocupa, el Community Manager, se ha hecho en base a cuatro vertientes, con el fin de cuantificar los contenidos relacionados con la figura ${ }^{18}$ :

\footnotetext{
${ }^{17}$ La medición de la audiencia en Internet comenzó en 1996 por parte de Asociación para la Investigación de Medios de Comunicación (AIMC); en ese año se estimó que en España había 342.000 internautas.

${ }^{18}$ La selección de estas páginas web y espacios online se ha basado en el ranking general de audiencia (Google), el ranking de portales de empleo (Infojobs e Infoempleo), el ranking de redes sociales (Facebook como red social generalista más importante y Linkedln como la principal red social profesional) y el ranking de portales especializados en marketing, todos ellos de la empresa Nielsen NetRatings (www.nielsen.com), empresa que lidera el mercado de la medición de la audiencia online.
}

Facultad de Ciencias de la Información - Universidad de La Laguna

Avenida César Manrique, s/n; Campus de Guajara

38071 La Laguna, Tenerife (Islas Canarias - España) 
- El buscador Google (www.google.com), como la página web con mayor tráfico en el ámbito español, con el objetivo de valorar el ruido que esta nueva figura profesional está generando.

- Los portales verticales de empleo Infojobs (www.infojobs.net) e Infoempleo (www.infoempleo.com), con el fin de conocer las ofertas profesionales que este perfil está creando.

- Facebook (www.facebook.com) y Linkedln (www.linkedin.com), con el propósito de conocer su presencia en estas redes sociales online.

- La plataforma de video digital Youtube (www.youtube.com), por ser una de las principales manifestaciones de la Web 2.0, líder en audiencia como portal de videos.

- Portales verticales especializados en marketing, comunicación y/o publicidad, para evaluar el impacto que la figura del Community Manager está teniendo en los contenidos de estos ámbitos profesionales. Estos portales son: Puro Marketing (www.puromarketing.com), Marketing News (www.marketingnews.es), El Publicista (www.elpublicista.es), Control (www.controlpublicidad.com), Interactiva Digital (www.interactivadigital.com), Anuncios (www.anuncios.com), IP Mark (www.ipmark.com), Brandlife (www.brandlife.es), Marketing Directo (www.marketingdirecto.com) y Estrategias de Marketing y Comunicación (www.estrategias.com).

La encuesta auto-administrada a responsables de AERCO se envió vía correo electrónico a José Antonio Gallego, presidente de la Asociación, y a Manuela Battaglini, responsable de Comunicación Interna. A estos dos profesionales se les enviaron las siguientes preguntas abiertas:

- ¿Con cuántos socios cuenta AERCO?

- ¿Cuál es su perfil profesional, en la mayoría de casos en cuanto a formación académica?

- ¿Cuál es su procedencia: agencia de medios, agencia de publicidad, anunciante, soporte, freelance, empresa de investigación de mercado, etc.?

\section{Marco teórico}

\subsection{Estrategias empresariales en la Web 2.0}

Internet se ha consolidado como el medio más consumido por los españoles, según indica el estudio "Mediascope Europe 2010" publicado por la European Interactive Advertising Association (EIAA) ${ }^{19}$. La Web 2.0 alude a la Red como un espacio social caracterizado por la conversación global y la participación. De esta manera, el usuario para de ser mero

\footnotetext{
${ }^{19}$ www.eiaa.net.
} 
consumidor pasivo de contenidos a generarlos, editarlos y compartidos con su comunidad, dando paso a un escenario en el que el internauta está en el centro del proceso ${ }^{20}$.

"Cuando se escriba la historia del periodismo digital será muy difícil ignorar el gran error que cometieron los medios y empresas de comunicación: pensar que Internet es un medio de difusión y publicación de contenidos en una sola dirección", afirma Richard Gordon, profesor de la Universidad Norhtwestern en el "Especial sobre redes sociales y periodismo" de la revista Nieman de Harvard ${ }^{21}$.

Como ya predijo el Manifiesto Cluetrain al afirmar que "los mercados son conversaciones"22, los espacios de la Web 2.0 permiten a las empresas participar en conversaciones y aportar contenido de interés para los usuarios, en un entorno favorable tanto para consumidores como para marcas. Afirma Dans (2010: 140) a propósito de este Neohumanismo:

"El comienzo del énfasis en la individualidad en las empresas coincide [...] con el fuerte desarrollo de herramientas como blogs y redes sociales que permiten una expresión más sencilla de la individualidad y la personalidad en la red".

De esta manera, los medios sociales se han convertido en uno de los espacios fundamentales en la interacción y la comunicación de las personas, empresas e instituciones. Prueba de ello es el ranking de palabras más buscadas en Google en 2009, por este orden: Tuenti (red social), Youtube (plataforma de videos) y Facebook (red social) ${ }^{23}$.

Los blogs, las plataformas de videos en Internet como Youtube y las redes sociales online son recursos a los que cada vez con más frecuencia acuden los anunciantes a la hora de elaborar sus estrategias publicitarias, gracias a las capacidades de Customer Relationship Management ${ }^{24}$ y marketing viral ${ }^{25}$ con que cuentan éstos, además de la posibilidad de fragmentar audiencias, personalizar el mensaje y llevar a cabo una comunicación multicanal.

\footnotetext{
${ }^{20}$ El poder del usuario en la era de la Web 2.0 es el que llevó a la revista Time a nombrar a You (Tú) la figura del año en 2006. También la publicación Ad Age reconocía poco después al Consumidor como la Agencia del Año, en parte gracias a haber visto reforzada su faceta de prescriptor a través de las herramientas de la Web 2.0.

${ }^{21}$ Disponible en: http://www.nieman.harvard.edu/reports.aspx?id=100058

22 Puede consultarse en: http://www.well-comm.es/wellcommunity/wp-content/uploads/cluetrain.pdf

23 Puede leerse la noticia completa en: http://www.que.es/tecnologia/200912011447-tuenti-facebook-youtube-palabrasmas.html

${ }^{24}$ Gestión de la relación con los clientes. La cultura del Customer Relationship Management (CRM) se centra en maximizar la información con que cuenta la empresa sobre los clientes, identificarlos y diferenciarlos e incrementar la cuota de los clientes mediante la construcción de relaciones duraderas y rentables, creando valor para ambas partes.

${ }^{25}$ El marketing viral es una estrategia que promueve actividades cuyo objetivo es que sean los propios usuarios quienes propaguen el mensaje o la información de que se trate, aprovechando redes de comunicación y relaciones sociales ya existentes entre los individuos, generalmente con el objetivo de lograr un conocimiento de marca elevado, que sea exponencialmente mayor a la exposición y recepción iniciales de dicho mensaje.
} 
En España superamos la media europea en cuando a acceso a redes sociales (un $55 \%$ frente al dato europeo del 53\%), ver vídeos y televisión por Internet (60\% frente al porcentaje europeo del $38 \%$ ) y el uso de la mensajería instantánea (53\% frente al $51 \%$ de los usuarios europeos), entro otros ${ }^{26}$.

Visibilidad, conectividad y herramientas son los tres pilares de la Web 2.0. Los medios sociales generan lazos emocionales con un consumidor activo que es, a su vez, productor de contenidos. Espacios como las redes sociales online reflejan opiniones y preocupaciones de los internautas que participan en ellas, como pueden serlo a propósito de productos, empresas y marcas.

En plataformas como Facebook, las marcas se han convertido en un actor más en el tejido de las relaciones: de hecho, alrededor de la mitad de los usuarios de la red declaran unirse y seguir las actividades de las marcas ${ }^{27}$. Algunas de las estrategias empresariales que las empresas ponen en marcha mediante la presencia en los medios sociales son:

- Orientación empresarial hacia el cliente. La Web 2.0 ha permitido el paso de una economía de mercado a una economía de las relaciones. Es la filosofía del client first (también conocida como Kaizen en Oriente), en la que el cliente es la principal variable de marketing que guía la toma de decisiones.

Las marcas buscan estrategias para conseguir el nivel más alto de lealtad del cliente, entendido como la prescripción del producto/marca. Se busca la satisfacción del cliente, como paso previo a la fidelización, con estrategias de retención que involucren al cliente en el proyecto empresarial, incrementen la rentabilidad de la empresa y consigan la lealtad del cliente.

La orientación empresarial hacia el cliente describe un cambio de mentalidad en el seno de la empresa estrechamente relacionado con la esencia y las potencialidades de las herramientas de la Web 2.0, y por eso es vital que la empresa gestione su reputación y conversación online de una manera profesional.

- Segmentación. Las posibilidades de afinidad para construir el vínculo entre marca y consumidor que ofrece el medio Internet favorece a la empresa el establecimiento de relaciones individualizadas.

\footnotetext{
${ }^{26}$ Fuente: Red.es.

27 Principal resultado de la segunda oleada del estudio "Observatorio de Redes Sociales" realizado por The Cocktail Analysis (www.tcanalysis.com).
} 
La segmentación de mercado pone de relieve las oportunidades de negocio existentes, al identificar grupos con demanda desatendida que pueden aumentar la participación de la empresa en el mercado, contribuye a establecer prioridades, facilita el análisis de la competencia, favorece el ajuste de la oferta a necesidades específicas y permite optimizar la inversión publicitaria.

Viralidad. Como hemos comentado en la Introducción, la recomendación de conocidos, conocido como boca-oreja, es el canal en el que más confían los consumidores en sus decisiones de compra y consumo.

Blogs, redes sociales online y videos digitales han hecho que el consumidor multiplique su faceta de prescriptor. El consumidor representa así un papel vital en las estrategias de los anunciantes dada su capacidad de intervención en los mensajes publicitarios gracias a la viralidad e interactúa con las marcas a través de los espacios de la Web 2.0.

Branding. Las comunicaciones personalizadas, interacciones y experiencias de marca que pueden ofrecerse en los medios sociales facilita la creación de comunidades en torno a la marca. Con estas nuevas herramientas, las empresas pueden relacionarse con los consumidores, aportarles valor y, por lo tanto, generar branding social.

En su intento de crear valor en la relación con sus públicos, la diferenciación de la empresa se da cuando los intangibles consiguen llegar al usuario a través de nuevos canales que permiten una relación más cercana con la marca, como son los medios sociales.

Un estudio llevado a cabo por la agencia Digital Nurun ${ }^{28}$ mostró que de todas las marcas en España registradas en Twitter, el 31\% no había interactuado nunca con los usuarios, de tal manera que se olvidaban de la finalidad de la red de microblogging y no aprovechaban las posibilidades que ésta les ofrece para acercarse a los consumidores e interactuar con ellos. Únicamente el $17 \%$ de las marcas había superado el centenar de conversaciones con usuarios. Por tanto, no sólo es necesario tener presencia en los medios sociales, sino también, como algo fundamental, darle continuidad y gestionar la comunicación a través de estos espacios con regularidad.

El poder absoluto otorgado a los usuarios en la Web 2.0 puede generar resultados inesperados para las empresas; las marcas deben estar preparadas ante las críticas que

\footnotetext{
${ }^{28}$ El estudio analizó a 175 marcas españolas con presencia en Twitter entre agosto de 2008 y noviembre de 2009 con el objetivo de estudiar la comunicación de las firmas en España con los usuarios de Twitter. Puede consultarse la noticia del estudio en: http://www.puromarketing.com/3/6815/las-marcas-estan-twitter-pero-no-trabajan-traves-red-social.html
}

Facultad de Ciencias de la Información - Universidad de La Laguna

Avenida César Manrique, s/n; Campus de Guajara

38071 La Laguna, Tenerife (Islas Canarias - España)

www.revistapangea.org | Pág. 83 
puedan afectar a su reputación y tener capacidad de previsión y reacción. Por todo ello se justifica la existencia del nuevo perfil que estamos estudiando, el Community Manager, como el profesional que va a gestionar y coordinar las comunidades y comunicaciones online de la organización.

Al abanico de posibilidades publicitarias ya existentes en los medios sociales (formatos publicitarios estándar, generación de contenidos como perfiles corporativos o aplicaciones, convocatorias a eventos y concursos, anuncios sociales, patrocinio de conversaciones online, etc.) se le suman nuevas derivadas de los continuos desarrollos tecnológicos, como por ejemplo la geolocalización ${ }^{29}$, que permite a los usuarios informar en todo momento de dónde se encuentran, incluidos establecimientos y comercios, en las plataformas digitales a través de sus dispositivos móviles.

\subsection{Definición y funciones de Community Manager}

La figura del Community Manager surgió en EE.UU., como el encargado de escuchar a la comunidad online, relacionarse con ella en nombre de la empresa, hacer llegar a su compañía lo que se dice de ella en el mundo digital para identificar amenazas u oportunidades e integrar estos espacios en las estrategias de negocio de la empresa. Todo ello con inmediatez y transparencia, utilizando un lenguaje cercano y ofreciendo contenidos relevantes.

Publicaciones como el New York Times cuentan ya con un perfil conocido como Social Media Editor: Jennifer Preston, una periodista con 25 años de experiencia, es la encargada de distribuir el contenido del periódico en las redes sociales. Estos gestores de comunidades digitales se encargan de que el contenido esté donde se encuentran los lectores: en los espacios de la Web 2.0. Preston es la persona que está detrás del usuario New York Times en Facebook, Twitter, Flicke, MySpace, etc., y también quien responde, conversa y pregunta a los internautas que comentan la información del periódico en Internet.

Conversar con la audiencia, escuchar y distribuir contenidos de la organización en los medios sociales son los principales cometidos del Community Manager. Si Internet nunca fue considerado un medio unidireccional, este nuevo perfil profesional le pone nombre y apellidos al tan ansiado feedback.

El Community Manager envía mensajes a través de plataformas como Facebook o Twitter, actualizan el estado de su corporación, revisa lo que se dice de su producto, mercado y

\footnotetext{
${ }^{29}$ Sistema por el cual la ubicación geográfica se puede comunicar a través de dispositivos móviles, de tal manera que se asigna una coordenada geográfica a la información para explotarla desde una herramienta informática. Una de las más comunes es Foursquare.
} 
competencia, ofrece contenidos exclusivos (pre-estreno de campañas publicitarias en redes sociales online, concursos y sorteos, entrevistas y chats con personal de la empresa, etc.), revisa las estadísticas de su perfil corporativo (tráfico de visitas, comentarios, participantes, etc.), monitoriza lo que se dice de la marca en la Red e invita a los usuarios a interactuar, entre otras tareas.

Según el estudio "Marketing en medios sociales" realizado por Territorio Creativo ${ }^{30}$ sobre este perfil profesional, 8 de cada 10 empresas usan los medios sociales para generar branding y aprueban su eficacia para conseguir notoriedad de marca. De ellas, el $51 \%$ de las empresas cuentan un Community Manager para gestionar y dinamizar su comunidad online. Las visitas conseguidas y número de seguidores o suscriptores son los indicadores más utilizados para medir esta efectividad.

De acuerdo con este estudio, el $84 \%$ de las empresas que realizan acciones de marketing en medios sociales considera que la agencia debe aportar la estrategia, apoyar al Community Managery, en menor medida, crear campañas y desarrollar aplicaciones. En cualquier caso, son pocos los Community Managers que realizan estas funciones a tiempo completo, siendo más frecuente que compatibilicen estas actividades con otras.

Otro estudio realizado por Puro Marketing ${ }^{31}$ saca como conclusión la tendencia creciente a contar con la experiencia de profesionales de este perfil. Según este estudio, el $27 \%$ de las empresas confían en los Community Managers para sus estrategias de Social Media Marketing. Sin embargo, el estudio también subraya que, a pesar de que existen empresas y marcas que no han dudado en incorporar nuevos profesionales con este perfil para mejorar la gestión de su visibilidad, reputación y sus estrategias de marketing en los medios sociales, todavía hay quienes dudan sobre la conveniencia de contar con ellos.

El estudio "2010 Social Media Marketing Industry Report"32, elaborado por Social Media Examiner mediante una encuesta entre más de 8.600 comerciantes y propietarios de pequeños negocios y empresas con una edad media de entre 40 y 49 años, destaca que más de la mitad de las pequeñas y medianas empresas invierten al menos seis horas semanales a diferentes actividades en los Social Media con fines comerciales o como parte de sus estrategias de marketing. Para este estudio, Twitter y Facebook son las herramientas más populares entre los comercios.

\footnotetext{
${ }^{30}$ www.territoriocreativo.es. La encuesta se realizó mediante cuestionario online autoadministrado distribuido en diferentes medios sociales, en las dos primeras semanas de 2010, con una muestra total de 600 empresas. Estudio disponible en: http://dl.dropbox.com/u/4905692/TC_Estudio_encuestaSMM_abril2010.pdf

31 Puede consultarse en: http://www.puromarketing.com/42/7224/el-27-empresas-confian-community-managers-para-susestrategias-social-media-marketing.html

${ }^{32}$ Disponible en: http://marketingwhitepapers.s3.amazonaws.com/SocialMediaMarketingReport2010.pdf
} 
Territorio Creativo y AERCO publicaron en noviembre de 2009 el libro blanco "La función del Community Manager"33, en el que se describen dos tipos de gestores de Community Managers: el gestor de comunidades online "ad-hoc", es decir, de aquellos entornos de los que la empresa es propietaria, y el gestor de la comunidad de la marca en el resto de plataformas y espacios de la Web 2.0. Este documento destaca que "las organizaciones deberían empezar a comprender que la gestión de los medios sociales comienza a ser una función en sí misma, y replantearse su organización desde la base".

El documento también incluye un completo listado de herramientas de la Web 2.0 que el Community Manager debe manejar, así como unas normas básicas desde el punto de vista deontológico para gestionar la comunidad online ${ }^{34}$. En cuanto al departamento al que debería adscribirse el Community Manager en la empresa, el libro blanco afirma que suele estar asociado al departamento más innovador de la empresa, en función también del perfil del profesional.

La Asociación Española de Responsables de Comunidad Online (AERCO) $)^{35}$ es una entidad sin ánimo de lucro que nació en 2008, compuesta por profesionales relacionados con las comunidades virtuales y que tiene la misión de representar y atender las necesidades de los responsables de comunidades online, con los objetivos de impulsar su desarrollo personal y profesional y lograr una proyección creciente y sostenible. AERCO ofrece cursos certificados de especialización para Community Managers.

Desde el punto de vista de la Asociación, las funciones y responsabilidades del gestor de la comunidad online pasan por escuchar, responder, informar, conectar y realimentar. AERCO define al Community Manager en su página web como:

"aquella persona encargada/responsable de sostener, acrecentar y, en cierta forma, defender las relaciones de la empresa con sus clientes en el ámbito digital, gracias al conocimiento de las necesidades y los planteamientos estratégicos de la organización y los intereses de los clientes. Conoce los objetivos y actúa en consecuencia para conseguirlos. Muy genéricamente, podríamos decir que un Community Manager es aquella persona que preserva la identidad digital de la compañía".

En definitiva, el Community Manager ha pasado a ejercer un rol estratégico en la organización, en tanto en cuanto coordina y gestiona las acciones de Social Media Marketing llevadas a cabo por la empresa. La aparición de profesiones como la del Community

\footnotetext{
${ }^{33}$ Disponible en: http://www.box.net/shared/pgur4btexi

${ }^{34}$ El libro blanco incluye también comentarios de Community Managers de importantes marcas. Puede consultarse una entrevista con Palmira Ríos, Community Manager de la red social Happing, de Coca-Cola, en el siguiente enlace: http://marccortes.blogspot.com/2009/02/que-hace-un-community-manager-nos.html

${ }^{35}$ www.aercomunidad.org
}

Facultad de Ciencias de la Información - Universidad de La Laguna

Avenida César Manrique, s/n; Campus de Guajara

38071 La Laguna, Tenerife (Islas Canarias - España) 
Manager en el entorno digital es una muestra del cambio de rol del consumidor en la Web 2.0, que ha obligado a las empresas a un replanteamiento de sus estrategias de comunicación.

\subsection{Perfil profesional del Community Manager}

El Community Manager es el nexo de unión entre la empresa y el público en Internet, gestiona la reputación online, posiciona a la organización en el espacio virtual, conversa con la audiencia y crea contenidos para compartirlos. Este profesional ha de tener las actitudes y aptitudes necesarias para gestionar la comunidad online de la empresa. Capacidad de escucha, transparencia, reflexión, actitud crítica, liderazgo y vocación son algunas de las características que debe reunir este perfil profesional.

Facebook, Twitter o Flickr se están convirtiendo en soportes integrados dentro del plan de marketing del anunciante y, por tanto, es importante que la figura del Community Manager también se integre en las estructuras, sistemas y procesos de la organización. Para ello, además, es vital que el Community Manager se sienta muy identificado con la empresa a la que representa en el mundo digital, conozca sus valores, necesidades, objetivos y planteamientos estratégicos para ser capaz de comunicarlos, independientemente de si forma parte de sus estructuras o es parte del equipo en la agencia de medios o de publicidad que gestiona la cuenta.

Analizar la información que recaba la empresa en los Social Media es también una de las tareas de este profesional, con el fin de generar conocimiento útil que pueda ser utilizado para la investigación de mercado, la puesta en marcha de programas de fidelización (Customer Relationship Management) y el desarrollo de nuevas líneas de negocio, por ejemplo.

Generar empatía y ser discreto son otras dos cualidades que ha de tener el Community Manager, puesto que su objetivo es conseguir que sus interlocutores se identifiquen con la empresa y comulguen con sus valores, siempre manteniéndose en un segundo plano. Además, la empatía no sólo es necesaria para con clientes actuales y potenciales, sino también con otro público que ha cobrado importancia en la Red: los prescriptores. Los bloggers se han convertido en un público clave para la empresa, dada la capacidad de influencia en los lectores que tienen los comentarios, como los relacionados con productos o marcas, que éstos publican en sus espacios virtuales.

Otras cualidades que debe reunir el gestor de comunidades online son la flexibilidad y la creatividad: la rapidez con la que se producen cambios en el ámbito de las nuevas tecnologías le obliga a estar adaptándose de manera continua y a buscar nuevas opciones 
originales y eficaces para las estrategias empresariales en cada una de las plataformas y espacios emergentes.

El libro blanco elaborado por Territorio Creativo y AERCO que hemos comentado en el apartado anterior habla de una actitud abierta, de servicio y accesible como cualidades del Community Manager, además de ser transparente, humilde, conector y early adopter, es decir, pionero en el uso de nuevas tecnologías y herramientas digitales. Con respecto a su formación académica, el marketing, la comunicación, la publicidad y la informática parecen ser los ámbitos de formación fundamentales para el Community Manager. Entre las aptitudes técnicas que, en opinión de AERCO y Territorio Creativo, ha de tener el Community Manager, se encuentran:

- conocimiento del sector de la empresa, para afianzar la credibilidad y la reputación.

- Conocimientos de marketing, publicidad y comunicación corporativa, para entender objetivos de negocio y alinear su actividad con los mismos.

- Buena redacción.

- Pasión por las nuevas tecnologías, por Internet y la Web 2.0.

- Creatividad, para generar cuota de taención entre la sobreabundancia de información.

- Experiencia en comunicación online.

En muchas ocasiones, el Community Manager también tiene la tarea de evangelizar internamente, es decir, ser prescriptor del medio digital y de las herramientas que la empresa tiene a su disposición en la Web 2.0 para explotarlas al máximo.

La campaña publicitaria de la marca de refrescos Fanta que se emitió en mayo de 2010 en televisión ha aumentado la popularidad de la figura del Community Manager ${ }^{36}$. En el anuncio, se animaba al público destinatario del mensaje publicitario a participar en un concurso de Fanta para convertirse en DJs, surferos o Community Managers. Las reacciones desde los profesionales de la comunicación online no se hicieron esperar y tanto desde AERCO como desde iniciativas personales se defendió la profesionalidad y la necesaria formación para ejercer de gestor de comunidades online, una función compleja y de gran relevancia para la empresa $^{37}$. En cualquier caso, lo que demostró esta campaña publicitaria es la popularidad que ha adquirido la figura del Community Manager en nuestro país.

\footnotetext{
${ }^{36}$ Puede verse el spot en el siguiente enlace: http://www.youtube.com/watch?v=Dk1HdluuioQ

${ }^{37}$ Pueden verse reacciones de bloggers en las siguientes direcciones:

http://mentezurda.com/2010/05/21/aprende-a-ser-surfer-dj-o-community-manager/
}

http://www.lacriaturacreativa.com/2010/05/fanta-surfer-dj-o-community-manager.html

En estos dos enlaces puede leerse la reacción de AERCO sobre la campaña publicitaria de Fanta:

http://www.aercomunidad.org/2010/05/23/sobre-el-anuncio-de-fanta-y-sus-cursos-de-community-manager/ http://www.aercomunidad.org/2010/05/24/conversacion-con-los-responsables-de-coca-cola-sobre-el-anuncio-de-fanta/ 


\section{Resultados}

En el apartado Marco teórico hemos desarrollado la información obtenida a través de la investigación documental de las publicaciones listadas en la metodología. Dada la actualidad del objeto de estudio, la investigación documental se ha centrado en publicaciones profesionales del ámbito del marketing, la comunicación y la publicidad, al no existir una reducida bibliografía académica relacionada con el tema de estudio.

En este apartado desarrollaremos los resultados del análisis de contenidos de la Web 2.0 relacionados con la figura profesional del Community Manager ${ }^{38}$ y de la encuesta autoadministrada a responsables de AERCO.

En cuanto al análisis de contenidos en la Web 2.0, en general, tanto el Community Manager como la Web 2.0, las redes sociales online o el Social Media Marketing ocupan gran cantidad de contenidos en Internet, al ser su medio de origen y tratarse de "metacontenido", es decir, contenido sobre los propios medios sociales online. Por ejemplo, la búsqueda en Google de los términos exactos "Web 2.0", "redes sociales" y "Social Media Marketing" arroja $37.700 .000,10.600 .000$ y 32.400 .000 resultados, respectivamente. Además, la blogosfera se ha nutrido de estos temas, con gran cantidad de blogs que tratan sobre ellos (el $14,9 \%$, el $11,3 \%$ y el $10,6 \%$ de los contenidos indexados por Google corresponden a blogs para estos tres conceptos, respectivamente).

Centrándonos en el concepto que estamos estudiando, a continuación incluimos una tabla con los resultados de búsqueda del término exacto "Community Manager" en cada uno de los espacios digitales listados en la metodología:

\begin{tabular}{|c|c|c|c|c|c|}
\hline Buscador & \multicolumn{2}{|c|}{ Portales de empleo } & \multicolumn{2}{c|}{ Redes sociales } & $\begin{array}{c}\text { Plataforma de } \\
\text { videos }\end{array}$ \\
\hline Google & Infojobs & Infoempleo & Facebook & LinkedIn & Youtube \\
\hline 1.820 .000 & 13 & 7 & 3.200 & 13.460 & 953 \\
\hline
\end{tabular}

De los resultados en Google generales en Google, 71.000 páginas web son de España y 405 son noticias (el 14,3\% de España). El perfil profesional de esta figura también es un debate tratado con amplia cobertura en la blogosfera: 208.000 resultados generales $(11,4 \%)$ y

\footnotetext{
${ }^{38}$ Las búsquedas de los conceptos clase que se desarrollan en este apartado se realizaron entre los días 26 y 27 de mayo de 2010
}

Facultad de Ciencias de la Información - Universidad de La Laguna

Avenida César Manrique, s/n; Campus de Guajara

38071 La Laguna, Tenerife (Islas Canarias - España) 
10.800 resultados en España (15,2\%) en Google corresponden a blogs. Por tanto, podemos decir que es un tema preferente entre los tratados en blogs en España, al representar esta figura en la blogosfera hispana un porcentaje mayor que en los resultados de la blogosfera general. Los videos de Youtube contienen, principalmente, entrevistas con Community Managers y/o conferencias profesionales sobre esta nueva figura.

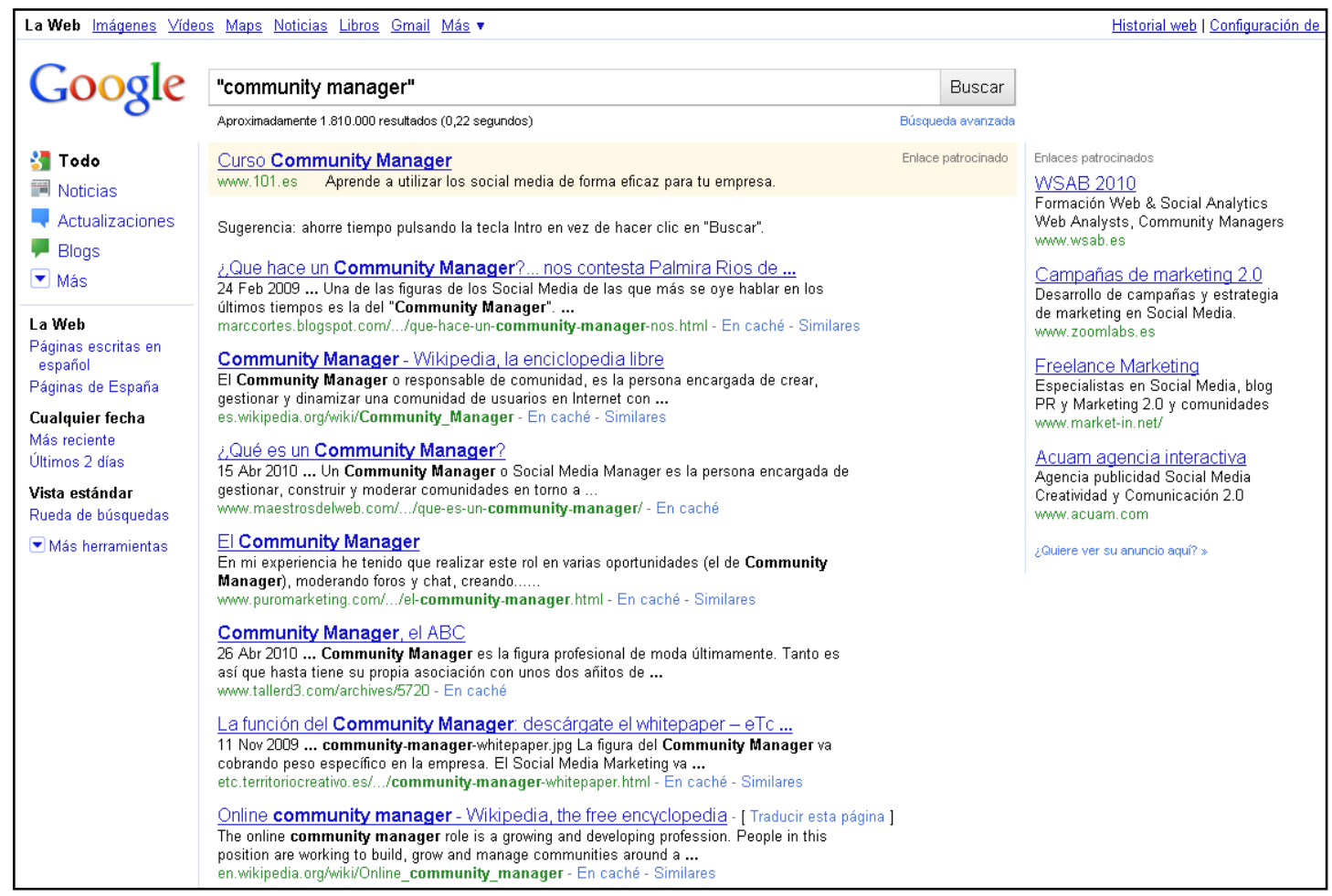

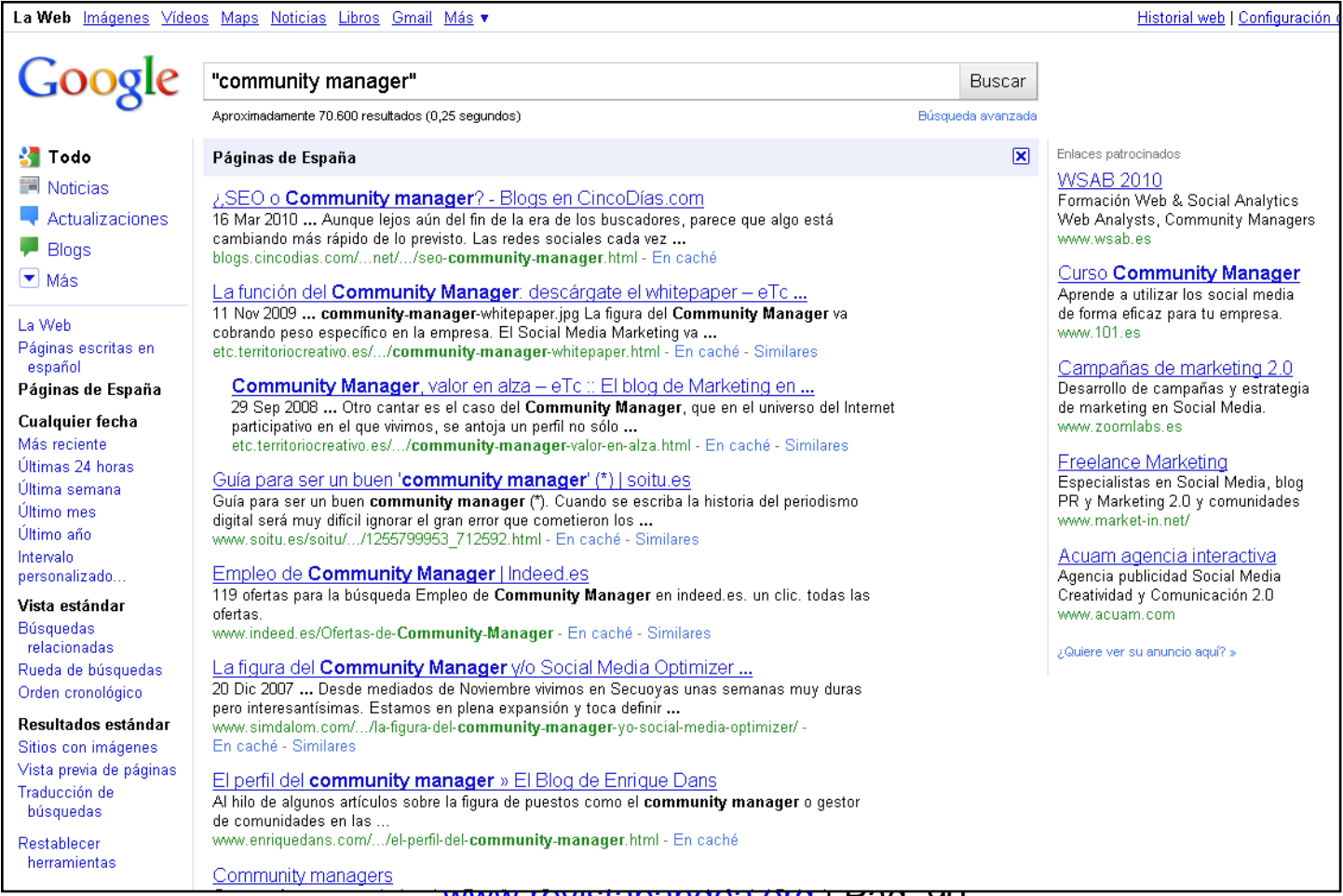


Los resultados de los portales de empleo Infojobs e Infoempleo se refieren a ofertas de trabajo en cuya descripción se incluye la figura del Community Manager. Incluimos a continuación las tablas de resultados con los criterios de búsqueda que ofrece Infojobs para las 13 ofertas encontradas:

\begin{tabular}{|lr|}
\hline \multicolumn{2}{|c|}{ Provincia } \\
\hline Barcelona & 5 \\
Madrid & 4 \\
Ciudad Real & 1 \\
Alicante & 1 \\
Segovia & 1 \\
Valencia & 1 \\
\hline
\end{tabular}
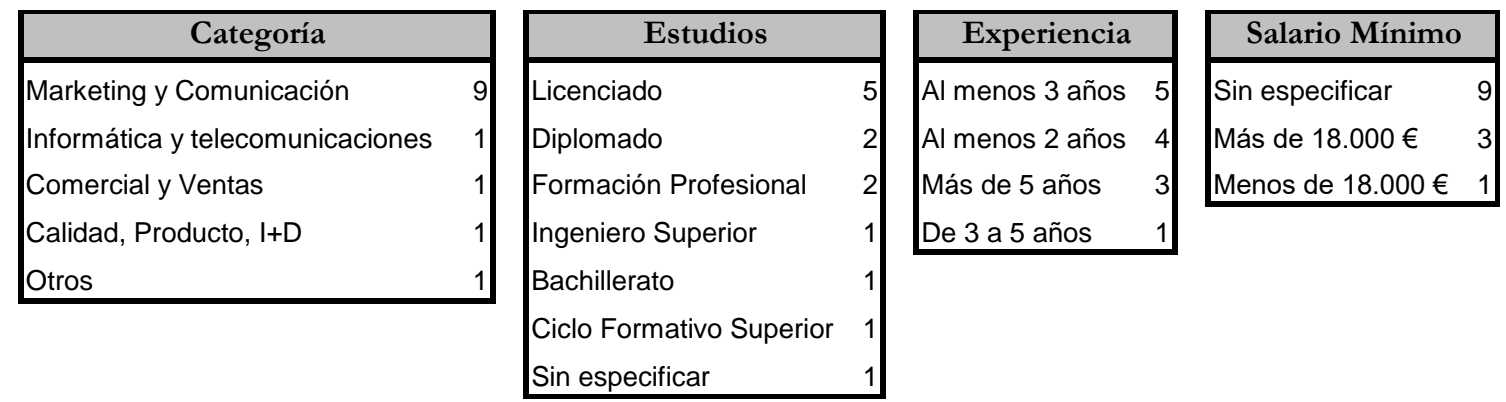

Como vemos, la mayoría de las ofertas (69\%) se dan en las provincias de Barcelona y Madrid. El perfil más demandado sería el de una persona con estudios superiores (38,4\% de licenciados), con formación en marketing y comunicación (69\%), con al menos 3 años de experiencia $(38,4 \%)$. En la mayoría de ofertas no se especifica el salario mínimo ofertado.

En cuanto a las redes sociales, en Facebook hemos obtenido 102 resultados de personas, más de 3.200 páginas, más de 500 grupos y 6 eventos en cuyos nombres aparece el término "Community Manager". Los eventos son conferencias, congresos y seminarios que tienen lugar todos en los próximos 15 días posteriores al día en el que se ha realizado la búsqueda.

En Linkedln, cabe destacar que el resultado de la búsqueda (13.460) son perfiles profesionales al tratarse de una red social profesional, es decir, son personas en cuyas descripciones profesionales aparece el concepto "Community Manager". Además, en esta red social se obtienen como resultado al buscar el concepto clave 26 grupos, de los cuales 11 son en español (42,3\%), con nombres como "Análisis estratégico y herramientas de colaboración web para el Community Manager", "Dirección de comunidades virtuales: el Community Manager como clave del éxito" del Instituto de Empresa o grupos locales ("Community Manager Barcelona" o "Community Manager Valencia").

Al ser Linkedln una red social profesional se ha convertido en una plataforma de reclutamiento y captación de profesionales para empresas, departamentos de recursos humanos y oficinas de headhunters. Prueba de ello es el aviso que apareció al realizar la búsqueda en la parte superior de los resultados, en el que Linkedln pregunta "¿Buscas candidatos de Community Manager? Anuncia tu empleo de Community Manager en LinkedIn", como vemos en la siguiente imagen: 
En

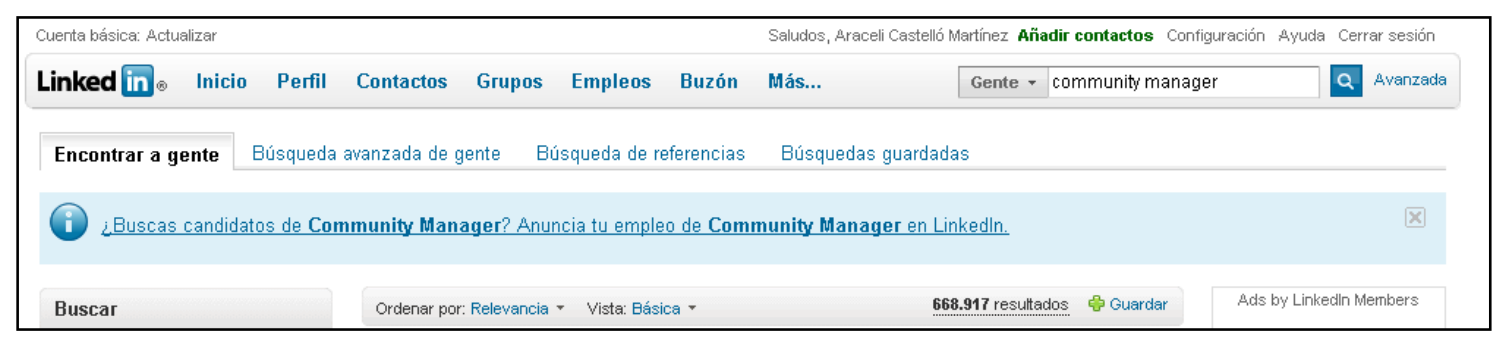

cuanto a las noticias aparecidas en portales verticales especializados en marketing, comunicación y/o publicidad que incluyen el concepto clave "community manager", los resultados han sido los siguientes:

\begin{tabular}{|c|c|c|}
\hline PORTAL & URL & RESULTADO \\
\hline Marketing Directo & www.marketingdirecto.com & 29 \\
\hline Marketing News & www.marketingnews.es & 10 \\
\hline Puro Marketing & www.puromarketing.com & 9 \\
\hline Interactiva Digital & www.interactivadigital.com & 7 \\
\hline Brandlife & www.brandlife.es & 3 \\
\hline Estrategias & www.estrategias.com & 3 \\
\hline El Publicista & www.elpublicista.es & 1 \\
\hline Control & www.controlpublicidad.com & 1 \\
\hline Anuncios & www.anuncios.com & 1 \\
\hline \multirow[t]{2}{*}{ IP Mark } & www.ipmark.com & 1 \\
\hline & TOTAL & 65 \\
\hline
\end{tabular}

En total, estas 10 publicaciones cuentan con 65 noticias en las que aparece el concepto de "Community Manager", relacionadas con seminarios, conferencias y congresos profesionales sobre los medios sociales, consejos profesionales para llevar a cabo una estrategia de Social Media Marketing o estudios como los que hemos mencionado en el marco teórico.

La encuesta auto-administrda a responsables de AERCO se envió vía correo electrónico a José Antonio Gallego, presidente de la Asociación, y a Manuela Battaglini, responsable de Comunicación Interna. A estos dos profesionales se les enviaron las siguientes preguntas abiertas:

- ¿Con cuántos socios cuenta AERCO? 
- ¿Cuál es su perfil profesional, en la mayoría de casos en cuanto a formación académica?

¿Cuál es su procedencia: agencia de medios, agencia de publicidad, anunciante, soporte, freelance, empresa de investigación de mercado, etc.?

AERCO cuenta con más de 1.200 socios, la mayoría de los cuales tiene formación en marketing, publicidad y/o comunicación. El $80 \%$ de los socios de AERCO pertenece a anunciantes o son freelance, por lo que son las agencias las que están inmersas en un proceso de incorporación a la asociación, sobre todo aquellas que cuentan con un departamento $\mathrm{o}$ un perfil profesional encargado de gestionar las comunidades online de sus clientes.

Manuela Battaglini destaca la labor de evangelización que tiene la asociación por delante, en tanto en cuanto la figura del Community Manager está creciendo muy rápidamente en número de profesionales dedicados a la tarea de gestionar las comunidades online y empresas conscientes de la importancia de este perfil profesional en las estrategias empresariales.

En opinión de los responsables de AERCO, el Community Manager ha de tener un perfil de marketing, identificando los intereses del cliente y alineándolos con las necesidades y estrategias de la empresa. Además de ser buen comunicador, esta figura ha de estar en contacto continuo y directo con las nuevas tecnologías y la Red y ser capaz de identificar nuevas oportunidades de negocio que puedan surgir para la empresa en la Web 2.0.

Según Juan Antonio Gallego, presidente de AERCO, lo idóneo es que el Community Manager sea alguien interno de la compañía, a nivel directivo, con capacidad de influencia interna y visión estratégica a largo plazo. Afirma, además, que la novedad del cargo hace que cada empresa coloque al gestor de comunidades online en un departamento diferente: marketing, publicidad, comunicación, informática, etc. Lo importante, según destaca este profesional, es que se trata un puesto estratégico.

Las publicaciones y estudios en los que hemos profundizado en el marco teórico, la cuantificación de la presencia del Community Manager en la Red y las opiniones aportadas por los responsables de AERCO demuestran la importancia que cada vez más conceden los anunciantes a esta figura, como profesional encargado de gestionar su comunicación online a través de las diferentes plataformas de la Web 2.0. Agencias de publicidad y de medios, soportes y anunciantes se encuentran en un proceso de incorporación a sus plantillas de personal cualificado para poder llevar a cabo estrategias de Social Media Marketing. 


\section{Conclusiones}

Como hemos desarrollado a lo largo del presente artículo, el progreso de nuevas tecnologías y formas de comunicación ha llevado a que Internet sea uno de los medios más consumidos por la población y utilizados por los anunciantes. De hecho, en países como el Reino Unido la inversión publicitaria en Internet ya ha superado a la destinada a la televisión, según datos del estudio sobre inversión publicitaria realizado por Interactive Advertising Bureau (IAB) y Pricewaterhouse Coopers ${ }^{39}$.

Ante este panorama, los anunciantes buscan instaurar sus estrategias de marketing y publicidad en el mundo digital. Las redes y medios sociales, la geolocalización mediante el acceso desde dispositivos móviles y el impulso del video online, entre otros, han dado origen a un nuevo escenario que requiere de una figura profesional que coordine todos los escenarios comunicativos online de la empresa: el Community Manager.

Segmentación, viralidad, branding y orientación empresarial hacia el cliente son estrategias a tener en cuenta en los planes de Social Media Marketing de las empresas, en los que la proximidad es la clave. Además, los medios sociales han permitido a pequeñas y medianas empresas llevar a cabo acciones de comunicación online, en las que obtienen notoriedad y contactos con clientes potenciales prácticamente a coste cero.

A medida que la figura del Community Manager vaya integrándose en las estructuras de las organizaciones (anunciantes, agencias de publicidad y de medios, soportes, etc.), los Social Media serán explotados no sólo como canales de información y comercialización, sino también de conversación.

La eclosión de las herramientas y plataformas sociales y su continuo desarrollo traerán consigo nuevos perfiles profesionales. Por ejemplo, el gestor de Social Media, entendido como una posición por encima del Community Manager y con unas funciones de planteamiento todavía más estratégico, el Chief Blogging Officer, responsable de los blogs de la compañía, el Customer Insight, que analizaría las necesidades de clientes actuales y potenciales, o el User Experience, encargado de velar por que los productos provoquen una experiencia positiva en el cliente. Todas ellas figuras que demuestran el poder que ha adquirido el estudio del consumidor, de su perfil sociodemográfico y psicográfico, desde el

\footnotetext{
${ }^{39}$ www.iab.co.uk. IAB es una asociación que opera a nivel internacional dedicada a temas de marketing y publicidad interactivos. Su fundación fue en EE.UU. en 1996, mientras que en Europa se instauró en 1998 y en España comenzó su andadura en 2001. Está presente en más de 20 países.
} 
punto de vista del neuromarketing ${ }^{40}$, así como de sus hábitos y comportamiento de compra, consumo y sociabilidad.

Una mala gestión del ruido de una marca en la Red puede generar desconfianza en el cliente. De ahí la importancia de crear vínculos fuertes con los consumidores, fidelizarlos y dialogar de una manera constante y solvente con ellos, tareas del Community Manager a través de las plataformas de la Web 2.0.

En definitiva, la web social está transformando la forma en que las empresas se relacionan con sus clientes, actuales y potenciales. Las empresas más innovadoras ya son conscientes de la necesidad de contar con este perfil profesional, que tanto ruido está generando en la Red, como hemos visto en el apartado Resultados.

Ello nos lleva a la necesidad de un estudio en profundidad sobre este perfil profesional desde el punto de vista académico, principalmente desde el ámbito de los sistemas y procesos de trabajo, con el fin de analizar su incorporación en el ámbito del marketing y la publicidad, su integración en las estructuras departamentales, su interacción con el resto de actores del mercado del marketing y la publicidad y su evolución a medio-largo plazo.

Podemos concluir que la figura del Community Manager está de moda, a la luz de los resultados obtenidos: los estudios sobre esta figura realizados desde el ámbito profesional, la cantidad de contenidos existente en la Red a propósito del gestor de comunidades online (congresos y cursos de formación, noticias, ofertas de trabajo, grupos de profesionales, etc.) y el crecimiento que en apenas dos años ha tenido la Asociación Española de Responsables de Comunidad Online (AERCO), son ejemplos de que la nueva oportunidad profesional que han generando los espacios de la Web 2.0 .

\section{Bibliografía}

Aced, C. (2010): Perfiles profesionales 2.0. Barcelona: Editorial UOC.

Alba, J. \& Stay, J. (2008): I'm on Facebook. Now what? Silicon Valley: Happy About.

\footnotetext{
${ }^{40}$ Neuromarketing es el estudio del funcionamiento del cerebro en las decisiones de compra de un producto. Se trata de un campo del marketing que investiga la respuesta cerebral a los estímulos publicitarios, de marca y de otro tipo de mensajes culturales, indagando en las zonas del cerebro que están involucradas en las acciones de compra y consumo de los consumidores.
} 
Benavides Delgado, J., et alt. (2008): Nuevas tendencias de la comunicación. Madrid: Universidad Complutense de Madrid.

Best, R. J. (2007): Marketing estratégico. Madrid: Pearson Educación.

Castelló Martínez, A. (2010): Estrategias empresariales en la Web 2.0. Las redes sociales online. Alicante: ECU.

Castelló Martínez, A. (2010): "La orientación empresarial hacia el cliente en la Web 2.0", en Miguel Hernández Communication Journal 1, España, Mayo: http://mhcj.es/2010/05/12/castello/

Celaya, J. (2008): La empresa en la Web 2.0. Madrid: Gestión 2000.

Dans, E. (2010): Todo va a cambiar. Tecnología y evolución: adaptarse o desaparecer. Madrid: Deusto.

De Salas Nestares, M.I. (2002): La comunicación empresarial a través de Internet. Valencia: Servicio de publicaciones Universidad CEU-Cardenal Herrera.

Estalella, A. (2007): "Teoría de los blogs. La construcción de la blogosfera: yo soy mi blog (y sus conexiones)", en VV.AA., La blogosfera hispana: pioneros de la cultura digital (Dir., Cerezo, J.M.). Madrid: Fundación France Telecom.

Evans, D. (2008): Social Media Marketing an hour a day. Indianapolis: Wiley Publishing, Inc.

Fumero, A. y Roca, G. (2007): Web 2.0. Madrid: Fundación Orange.

Gil, V. y Romero, F. (2008): Crossumer. Claves para entender al consumidor español de nueva generación. Barcelona: Gestión 2000.

Holzner, S. (2008): Facebook marketing: leverage social media to grow your business.

Nafría, I. (2008): Web 2.0: el usuario, el nuevo rey de Internet. Barcelona: Gestión 2000 D.L.

Ohmae, K. (2004): La mente del estratega. Madrid: McGraw-Hill.

Paul Gillin, G. (2007): The new influencers: a marketer's guide to the new social media.

Ros, V. (2008): E-branding: posiciona tu marca en la Red. A Coruña: Netbiblo, D.L.

Safko, L. \& Brake, D.K. (2009): The Social Media Bible. New Jersey: John Wiley \& Sons Inc. 
Shih, C. (2009): The Facebook era. Boston: Pearson Education.

Weinberg, T. (2009): The new community rules: marketing on the social web. Sebastopol: O’Reilly.

\section{Forma de citar este artículo en bibliografías}

Araceli Castelló Martínez (2010): “Una nueva figura profesional: el Community Manager”, en Revista PANGEA, 1, páginas 74 a 97. Red Académica Iberoamericana de Comunicación. Recuperado el _ de ___ de 2 __ de: http://revistapangea.org/2010/09/13/01$\underline{01-104 /}$ 